\title{
Human umbilical cord mesenchymal stem cells express cholinergic neuron markers during co-culture with amniotic membrane cells and retinoic acid induction
}

\author{
Mohammad-Reza Kouchakian ${ }^{1}$, Morteza Koruji ${ }^{1,2}$, Mohammad Najafi ${ }^{3}$, Seyedeh Farzaneh Moniri ${ }^{4}$, Alireza Asgari ${ }^{5}$, \\ Marjan Shariatpanahi ${ }^{6}$, Seyed Akbar Moosavi ${ }^{7}$, Hamid Reza Asgari ${ }^{1,2,7 *}$ (D)
}

Received: 23 Apr 2020

Published: 5 Oct 2021

Abstract

Background: A wide variety of cytokines are released from human amniotic membrane cells (hAMCs), which can increase the rate of differentiation of mesenchymal stem cells into the neurons. We studied the effect of Retinoic Acid (RA) on the differentiation rate of human Umbilical Cord Mesenchymal Stem Cells (hUMSCs) which were co-cultured with hAMCs.

Methods: In this experimental study, both hUMSCs and hAMCs were isolated from postpartum human umbilical cords and placenta respectively. The expression of mesenchymal (CD73, CD90 and CD105), hematopoietic and endotheliall (CD34 and CD45) markers in hUMSCs were confirmed by flow cytometry. The hUMSCs were cultured in four distinct groups: group 1) Control, group 2) Coculture with hAMCs, group 3) RA treatment and group 4) Co-culture with hAMCs treated by RA. Twelve days after culturing, the expression of NSE, MAP2 and ChAT differentiation genes and their related proteins were examined by real-time PCR and immunocytochemistry respectively.

Results: The flow-cytometry analysis indicated increased expression of mesenchymal markers and a low expression of both hematopoietic and endothelial markers (CD73:98.24\%, CD90: 97.32\%, CD105: 90.75\%, CD34: 2.96\%, and CD45:1.74\%). Moreover, the expression of both NSE and MAP2 markers was increased significantly in all studied groups in comparison to the control group On the other hand, the expression of ChAT had a significant increase in the group 2 and 4 (RA and RA+ co-culture).

Conclusion: RA can be used as an effective inducer to differentiate hUMSCs into cholinergic-like cells, and hAMCs could increase the number of differentiated cells as an effective factor.

Keywords: Amniotic Membrane, Cell Differentiation, Mesenchymal Stem Cell, Neurons, Retinoic acid

Conflicts of Interest: None declared

Funding: This study was funded by a grant from Iran University of Medical Sciences (IUMS), (Number: 95-01-30-27942) for an M.SCs student thesis.

\section{*This work has been published under CC BY-NC-SA 1.0 license.}

Copyright $₫$ Iran University of Medical Sciences

Cite this article as: Kouchakian MR, Koruji M, Najafi M, Moniri SF, Asgari A, Shariatpanahi M, Moosavi SA, Asgari HR. Human umbilical cord mesenchymal stem cells express cholinergic neuron markers during co-culture with amniotic membrane cells and retinoic acid induction. Med $J$ Islam Repub Iran. 2021 (5 Oct);35:129. https://doi.org/10.47176/mjiri.35.129

Corresponding author: Dr Hamid Reza Asgari, asgari.hr@iums.ac.ir

1. Department of Anatomy, Faculty of Medicine, Iran University of Medical Sciences, Tehran, Iran

2. Stem Cell and Regenerative Medicine Research Center, Iran University of Medical Sciences, Tehran, Iran

3. Department of Biochemistry, Faculty of Medicine, Iran University of Medical Sciences, Tehran, Iran

4. Department of Anatomy, School of Medicine, Tehran University of Medical Sciences, Tehran, Iran

5. School of Pharmacy, Zanjan University of medical sciences, Zanjan, Iran

6. Department of Toxicology \& Pharmacology, School of Pharmacy, International Campus, Iran University of Medical Sciences, Tehran, Iran

7. Cellular and Molecular Research Center, Iran University of Medical Sciences, Tehran, Iran

\section{$\uparrow$ What is "already known” in this topic:}

Given the prevalence of nervous system diseases, scientists plan to differentiate stem cells into neurons and use them in the treatment of neurodegenerative diseases. In laboratory studies, different stem cells and inducers have been used to differentiate neurons. Cord-derived mesenchymal stem cells are readily available and have a high differentiation potential. Amniotic membrane cells secrete growth factors suitable for differentiation, and retinoic acid is also a good inducer of stem cell differentiation into neurons.

\section{$\rightarrow$ What this article adds:}

In this study, umbilical cord stem cells under RA induction and during amniotic cell co-culture showed a significant increase in the expression of NSE and MAP2 neuronal markers in all groups compared to the controll group. While in the expression of the specific marker of cholinergic neurons, ChAT in the coculture group, in addition to retinoic acid, also had a significant increase. 


\section{Introduction}

Nowadays, regenerative medicine researchers are trying to have exploited the potential of stem cells to treat various neurodegenerative diseases. The underlying treatment base was to compensate for tissue defects in various disease $\mathrm{s}$ such as neurodegenerative diseases (1, 2). Since effective treatment does not exist for the diseases and stem cell therapy opens a new window for the treatment of neurodegenerative diseases.

Neural stem cells (NSCs) are the cells located in subventricular zone, sub-granular zone, olfactory bulb and hypothalamus $(3,4)$, with a capability of differentiation into neurons, astrocytes and oligodendrocytes (5). However, there are technical and ethical obstacles that have limited the NSCs isolation. Therefore, mesenchymal stem cells are suitable candidates for stem cells that can be obtained from bone marrow, adipose tissue, and umbilical cord (UC). Human umbilical cord mesenchymal stem cells (hUMSCs) are a young population of stem cells with the ability to differentiation into various cell lineages including mesodermal (osteoblast, adipocyte, chondrocyte), endodermal ( hepatocyte and pancreatic cells) and ectodermal cells (neuron, glial and epidermal cells) $(6,7)$. In addition, they lack the expression of HLA $\Pi$ and do not stimulate immune system response. Despite the low expression of pluripotency factors such as OCT4, Nanog, SOX2, TRA 1-60, TRA 1-81, SSEA4 and embryonic surface antigen marker, these cells may not induce teratoma formation in mice with immune deficiency but they express mesenchymal markers like CD90, CD73 and CD105 but lack of hematopoietic and endothelial markers (8-11).

Amniotic membrane (AM), the innermost layer of the placenta, with a thin and avascular membrane that is composed of amniotic epithelial cells and amniotic mesenchymal stem cells $(12,13)$. These cells secret various cytokines such as VEGF, HGF, EGF, KGF, TGFb, NT-3, NGF, BDNF, and bFGF that play an important role in plasticity and neural differentiation (14-16).

Retinoic acid (RA) is one of the derivatives of vitamin A that plays a crucial role in embryogenesis, such as the normal growth and evolution and differentiation of cells into neurons in different tissues, especially in the central nervous system. Furthermore, RA has an important task in adult stem cell proliferation and regeneration as well as axon healing after neural injury $(17,18)$. On the other hand, it can be lethal and toxic to the cells in higher concentrations. However, the medium of the co-culture system has some growth factors that can reduce the level of toxicity (19).

In this study, we investigated the effect of co-culturing hAMCs with RA on the differentiation of hUMSCs into the cholinergic-like cells by indirect co-culture.

\section{Methods}

\section{Isolation of hUMSCS}

In this experimental study, after receiving parental consent and evaluation of the babies in terms of AIDS and Hepatitis, three fresh human umbilical cords were collected from full-term births with cesarean delivery and placed in Hank's Balanced Salt Solution and immediately transferred to the laboratory. Tissues were washed using sterile PBS with $1 \%$ Penicillin-Streptomycin (P/S) [Gibco, Waltham, USA] and cut to 3 to $5 \mathrm{~cm}$ pieces. After removing vessels, the jelly-like tissue was cut into small pieces, transferred to a T25 cell flask and cultured with DMEM-F12 with 10\% FBS (Medium) [Gibco, Waltham, USA]. Tissue species were harvested after the formation of the first mesenchymal cells colony within 10-20 days. (This study was conducted with code of ethics No. IR.IUMS.REC. 1395.27942.)

\section{Isolation of AM Cells}

Three fresh placentas were transferred to molecular and cellular research center. AM was detached from the chorionic membrane and placed in a sterile petri dish and cut into small pieces and then blood and clots were removed and washed off with sterile PBS and $1 \%(\mathrm{P} / \mathrm{S})$. Enzymatic digestion was performed initially with $1 \%$ Collagenase type I [Gibco, Waltham, USA] to isolate amniotic mesenchymal stem cells through incubation at $37^{\circ} \mathrm{C}$ and $5 \% \mathrm{Co} 2$ for 1 hour. This followed by second digestion to isolate amniotic epithelial cells using $0.25 \%$ trypsin EDTA [Gibco, Waltham, USA] and incubated again for two minutes (20). The enzymatic process was neutralized by the addition of a culture medium. The resulted pieces were removed from the tube and centrifuged at $1200 \mathrm{rpm}$ for $5 \mathrm{~min}$ at room temperature. The supernatant was discarded and cell pellets resuspended by fresh medium and transferred to T25 cell culture flask for culturing purpose.

\section{Cell culture}

Both hUMSCs and hAMCs were cultured in the appropriate medium. The cells were incubated at $37^{\circ} \mathrm{C}$ in $5 \% \mathrm{Co} 2$ gas and then harvested with $0.25 \%$ TrypsinEDTA after confluency of $70-80 \%$.

\section{Flow cytometry analysis}

Flow cytometry was used for the characterization of hUMSCs. First, cells were detached by Trypsin-EDTA $0.25 \%$ in the third passage and centrifuged at $1200 \mathrm{rpm}$ for 5 min. Then, $10^{5}$ cells were resuspended in PBS and incubated with monoclonal antibodies against CD-34PE, CD45-FITC, CD73-PE, CD105-FITC and CD90-FITC markers[Abcam, Cambridge, United Kingdom] for $20 \mathrm{~min}$ at room temperature. Isotype antibodies conjugated to FITC or PE were used for cell surface staining. Finally, the cells were fixed with $1 \%$ paraformaldehyde solution (PFA) and analyzed by Flomax software.

\section{Feeder cells culture}

The proliferation of hAMCs, were inactivated in passages 3 using Mitomycin-C as described (6). To provide an indirect co-culture system, hAMCs were dissociated by trypsin-EDTA $0.25 \%$ and seeded on Thinsert with $0.4 \mu$ pores (Greiner, Kremsmünster, 
Austria) using DMEM-F12 medium with 5\% FBS.

Differentiation process hUMSCs were also dissociated by Trypsin-EDTA $0.25 \%$ after third passages and seeded in 6 well plate and cultured with the medium. hUMSCs were set in four groups including group 1, control group, group 2, treated by RA, group 3, co-culture with hAMCs and group 4, hUMSCs treated by RA and co-cultured with hAMCs. In order to differentiate, the cells were cultured in DMEM-F12 and 5\% FBS for 12 days. To establish a co-culture system, inserts containing hAMCs were placed on 6 well plates that contained hUMSCs, and intreated groups, cells were treated with $1 \mu \mathrm{M} / \mathrm{ml}$ RA.

\section{Immunocytochemistry analysis}

12 days after treatment with RA or co-culture, hUMSCs were rinsed with PBS and fixed in 4\% PFA for $10 \mathrm{~min}$ at room temperature.

In order to accomplish permeabilization, cells were washed with PBS 3 times and incubated in $0.1 \%$ Triton X100 [Sigma, Missouri, United States] for $10 \mathrm{~min}$. For blocking non-specific binding sites, cells were incubated in blocking solution, including PBS with 5\% goat serum for $45 \mathrm{~min}$ at room temperature. Afterward, cells were incubated with anti-NSE, anti-MAP2, and anti-ChAT antibodies [Abcam, Cambridge, United Kingdom] overnight at $4^{\circ} \mathrm{C}$, followed by incubation with Goat AntiRabbit FITC [Abcam, Cambridge, United Kingdom] in PBS with $1 \%$ FBS for 1 hour at room temperature. For crosslinking antigen/antibody complexes, cells were washed with PBS and fixed with 4\% PFA for $5 \mathrm{~min}$. Finally, cell nuclei were counter-stained with Propidium iodide (PI) [Sigma, Missouri, United States] for three minutes and examined under a fluorescent microscope (Olympus, IX71).

\section{Real-time PCR analysis}

RNA isolation was done by RNeasy mini kit [Qiagen,
Hilden, Germany] according to its handbook instruction. RNA purity and concentration were determined by the spectrophotometric method (Thermo fisher one plus, life science). The cDNA synthesis was made by QuantiNova Reverse Transcription Kit [Qiagen, Hilden, Germany] according to the instructions. cDNA concentration and purity were determined by the spectrophotometric method. Genes expression were evaluated by primers including MAP2 (forward 5' GGAACCAACTCTCTCTGGATTT 3'- reverse 5' GCATTCTCTC'TTCAGCCTTCT 3'), NSE (forward 5' GGACTATCCTGTGGTCTCC 3'- reverse 5' CTACATTGGCTGTGAACTTG 3') ChAT (forward 5' CCACTCCATTCCCACTGACT 3'- reverse 5 GAGTGAACAGATCCCCCTCA $3^{\prime}$ ) and $\beta$-ACTIN (forward 5' TCCCTGGAGAAGAGCTACG 3'-reverse 5' GTAGTTTCGTGGATGCCACA 3'). To quantify the gene expressions, real-time PCR reaction was prepared by combined forward primer $(0.5 \mu \mathrm{l})$, reverse primer $(0.5 \mu \mathrm{l})$, cDNA template $(1 \mu \mathrm{l})$, Sybergreen $(5 \mu \mathrm{l})$ [Takara, Japan] and RNase free water $(3 \mu \mathrm{l})$. Real-time PCR parameters were set for $10 \mathrm{~min}$ at $94^{\circ} \mathrm{C}$ and 40 cycles of $94^{\circ} \mathrm{C}$ for 5 seconds and $58^{\circ} \mathrm{C}$ for 30 seconds using a Rotor-gene $\mathrm{Q}$ instrument. Relative expressions of target genes were calculated using the $\Delta \Delta \mathrm{Ct}$ method.

\section{Statistical analysis}

Data presented in this study had mean \pm standard deviation. Results for between-group comparison were tested with an independent t-test and one-way ANOVA. A $\mathrm{p}<0.05$ was considered statistically significant.

\section{Results}

\section{Cells morphology}

Cell colonies were observed in flasks 20 days after cultivation. Morphologically, hUMSCs had fibroblastic phenotype and revealed spindle shape with both long and short cell processes (Fig. 1).
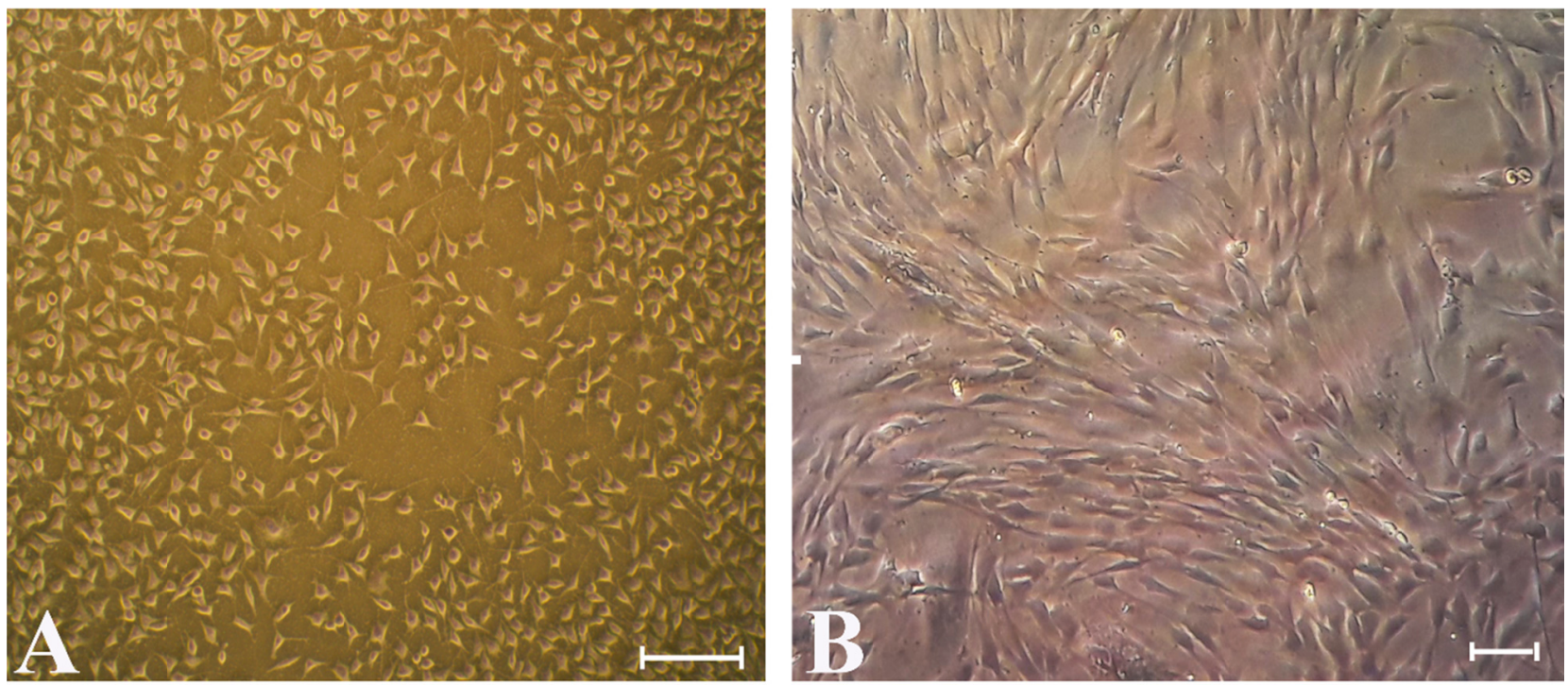

Fig. 1. hUMSCs morphology by invert microscopy. The proliferative state [Scale Bar $100 \mu \mathrm{m}]$ (A) and confluent state in the third passage [Scale Bar $20 \mu \mathrm{m}$ ] (B). hUMSCs have spindle-like shape with long and short process. 


\section{Flow cytometry findings}

hUMSCs were assessed by the flowcytometry method. Expression of mesenchymal markers for CD73, CD90 and CD105 was $98.24 \%, 97.32 \%$ and $90.75 \%$, respectively. While the cells expressed $2.96 \%$ and $1.74 \%$ of hematopoietic and endothelial markers (CD34 and CD45) respectively (Fig. 2), these results indicated that hUMSCs formed a high population of mesenchymal stem cells.

\section{Real-time PCR analysis for gene expression}

Real-time PCR measured the expression of neural genes including NSE, MAP2, and ChAT in treated cells and the results were compared with the control group. In group 3 (co-culture), the expression of NSE (3.15) and MAP2
(3.83) significantly increased $(\mathrm{p}<0.01)$, while the expression of ChAT (1.16) did not change significantly. Moreover, in group 2 (RA), the expression of NSE, MAP2 and ChAT were $2.47,4.25$ and 3.50 respectively indicated a significant increase in all three groups of treatment compare to the control group $(\mathrm{p}<0.05)$. In both groups 2 and 3 (RA and co-culture), there was also a significant increase in expression of NSE, MAP2 and ChAT in comparison to the control group $(p<0.001$ for MAP2, NSE and $p<0.01$ for ChAT). Nonetheless, ChAT expression increased significantly in group 2 and 4 (RA group and RA+ co-culture group) while in group 3 (co-culture), the expression quantity of ChAT had no significant increase in contrast to the control group (Fig. 3).
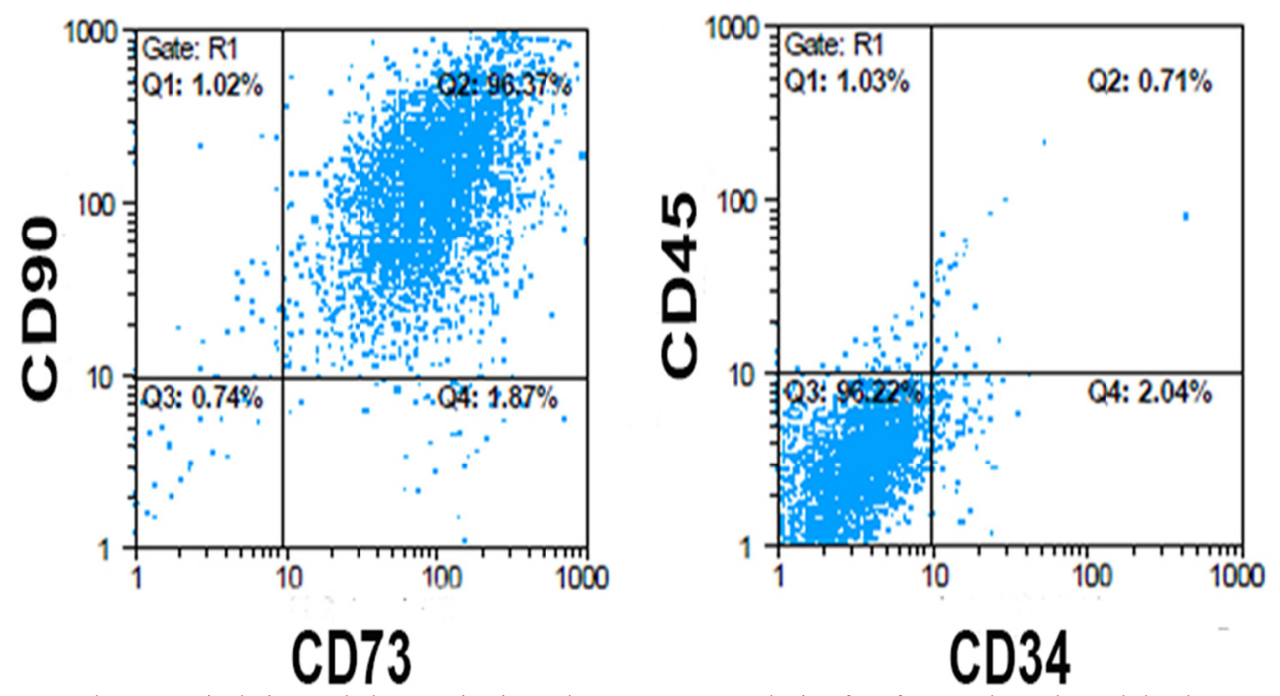

Fig. 2. hUMSCs isolation and characterization: Flowcytometry analysis of surface markers showed that hUMSCs express high level (positive) of CD90 \&CD73 and low level (negative) of CD34 \& CD45.

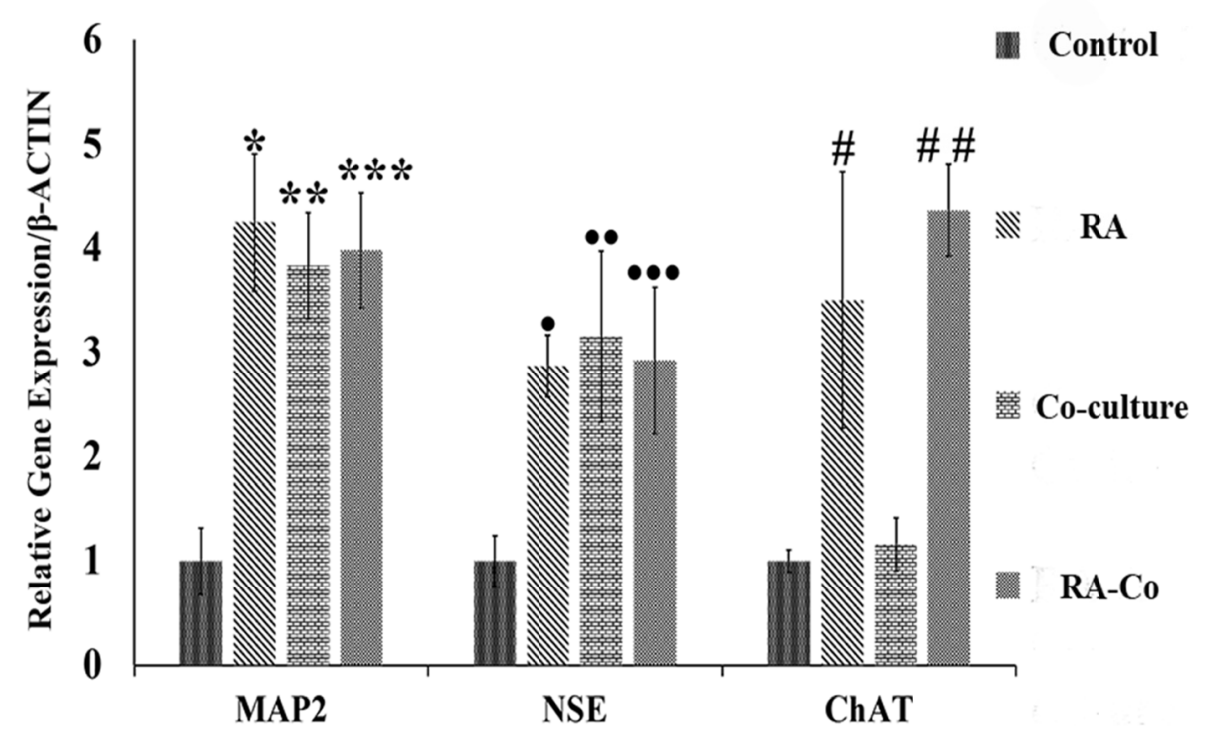

Fig. 3. Real-Time PCR results indicating the expression of neural markers by using RA and co-culturing with hAMCs. The expression of MAP2 and NSE markers increased significantly compared to the control group. Each point explains Mean \pm S.E.M. (*,** and *** indicates P- values $0.02,0.044$ and $0.041 \pm \mathrm{SD}$, respectively. $\bullet, \cdot \bullet, \cdots$ shows $\mathrm{P}$ - values $0.05,0.038$ and $0.034 \pm \mathrm{SD}$, respectively). ChAT expression did not change significantly in co-culture group comparing to the control group $(\mathrm{P}$ - value $=0.808 \pm \mathrm{SD})$ while it increased significantly in RA group and RA \& Coculture groups in comparison with control group (\# indicates $\mathrm{P}$-value $=0.04 \pm \mathrm{SD}$ and \#\# shows $\mathrm{P}$ - value $=0.027 \pm \mathrm{SD}$ ). 


\section{Immunocytochemistry differentiation analysis}

The in vitro neural differentiation of hUMSCs was assessed by immunocytochemistry analysis, indicated the presence of NSE and MAP2 in all treated groups, while ChAT expression was seen in group 2 and 4 (RA group and $\mathrm{RA}+$ co-culture group), but no expression found in co-culture group (Fig. 4).

\section{Discussion}

After the discovery of stem cells, scientists have been tempted to use stem cells to pave the way for the treatment of neurodegenerative diseases $(21,22)$. Among different types of stem cells, human umbilical cord mesenchymal stem cells have been established to be a superior option to be used in regenerative medicine and cell therapy owing to its non-invasiveness, high proliferation rate and differentiation capacity as well as ability to differentiate into different cell lines (23).

Nevertheless, the differentiation capacity of these cells to various types of neuronal cells has been proved, and it is shown to be effective for recovering the tissue damage in the nervous system (24). A variety of techniques e. g. using inducers, cytokines, co-culturing, and genetic methods, have been used to differentiate stem cells into neu- rons.

Studies show that RA, as a specific ligand for the retinoic acid receptor (RAR), has many functions, including the development of the nervous system (due to characteristic neuronal differentiation), axonal growth, protection of adult neurons and neural stem cells (18). Thus it has been applied alone or in combination with other inducing agents to differentiate various types of stem cells into neurons (25-27). Javeri et al. (2014) showed that the effect of RA treatment on neural differentiation in rat adipocyte mesenchymal stem cells during two weeks of cultivation. Although $1 \mu \mathrm{M} / \mathrm{ml}$ of RA did not change the expression of the NSE gene (Neural Specific Enolase) significantly, the expression level increased by 1.5 fold with 0.1 and $0.01 \mu \mathrm{M} / \mathrm{ml}$. On the other hand, no change in the expression of ChAT gene was observed in any of the groups (28). In our study addition of $1 \mu \mathrm{M} / \mathrm{ml}$ of RA to the culture medium increased the expression of NSE and ChAT by 2.5 and 3 fold, respectively. This could be explained by the difference in cell types and possibly the higher ability of the hUMSCs for differentiating into the neurons (29). The higher amounts of RA can reduce the cellular life while in low concentrations can improve the survival of differentiating stem cells (24). In another study conducted

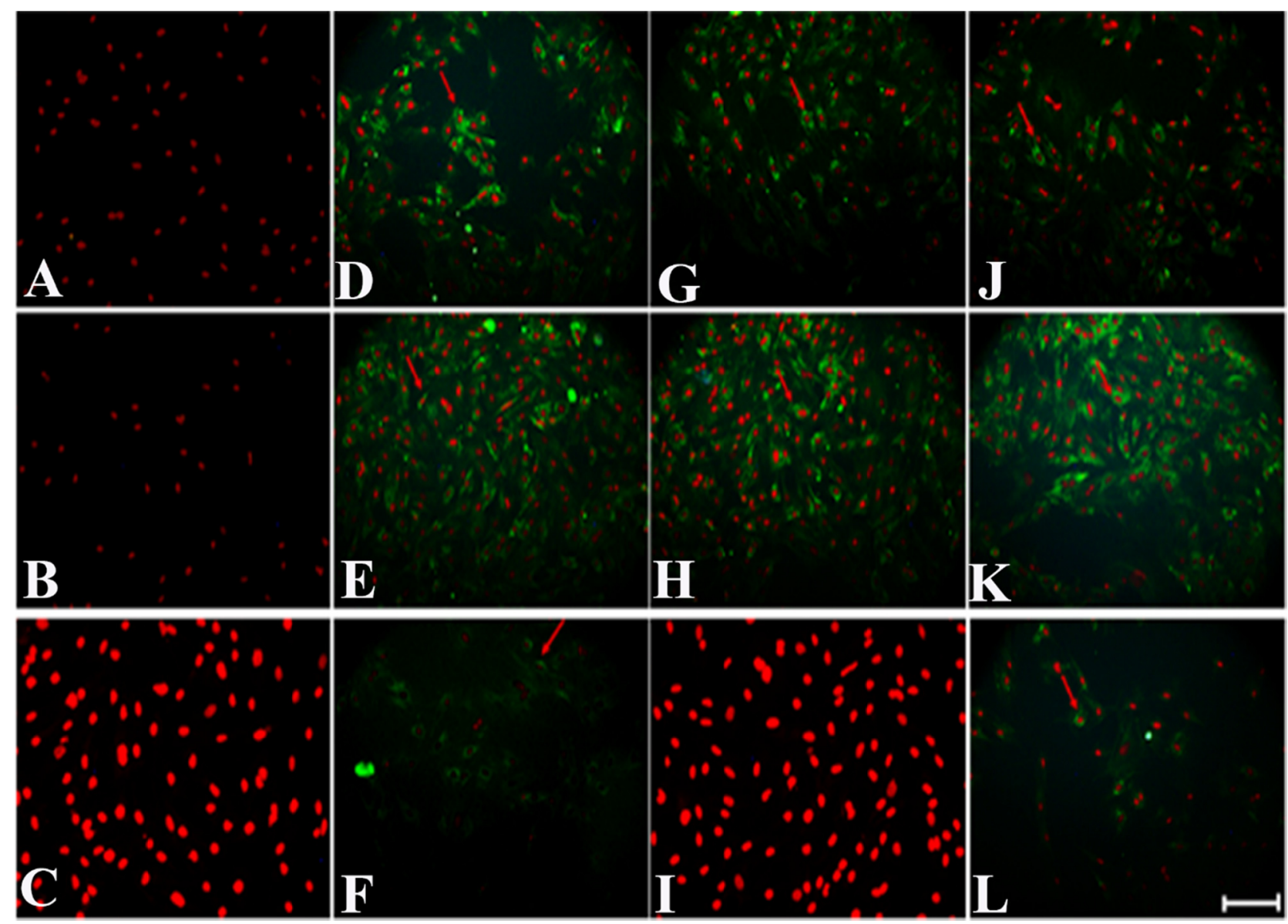

Fig. 4. Immunocytochemistry of neural markers in human umbilical cord mesenchymal stem cells after 12 days' treatment in different experimental groups including: Control group (A: NSE, B: MAP2, C: ChAT), Retinoic Acid group (D: NSE, E: MAP2, F: ChAT), Co-culture group (G: NSE, H: MAP2, I: ChAT) and Retinoic Acid with Co-culture (J: NSE, K: MAP2, L: ChAT). Green colors indicate the expression of neural protein markers such as NSE, MAP2 and ChAT that defined by red arrows. Nuclei were stained with PI and presented in red color (Scale Bar: $20 \mu \mathrm{m})$. 
by Marei et al. (2018) it was revealed that $10 \mu \mathrm{M} / \mathrm{ml} \mathrm{RA}$ could increase ChAT expression by twofold in adiposederived stem cells (30). Whereas, in present study we showed that $1 \mu \mathrm{M} / \mathrm{ml}$ not only increased the expression of both NSE and MAP2 in hUMSCs but also increase the expression of ChAT (Cholinergic Neuron marker) compared to the control group.

The results of this study are compatible with Hynek Wichterle's theory, which depicted the role of RA in enhancing motor neurons and causing differentiation in a variety of motor neuron cells (31). Most of the scientists used retinoic acid as an effective inducer, while we achieved better results by using lower doses of retinoic acid due to the use of high potential hUMSCs. Previous studies found that AM consists of epithelial and mesenchymal stem cells and the membrane used as a biological scaffold to improve burns and corneal ulcers.

Mesenchymal stem cells of AM express various cytokines and growth factors, including EGF, TGF- $\beta$, KGF, HGF, bFGF, VEGF and TGF- $\alpha$. Likewise, the epithelial cells of the amniotic membrane secrete some other elements like NGF, BDNF and NT-3. In addition, factors secreted by adjacent cells can affect the fate of the stem cells. The co-culture system can create a cross-link between two groups of cells (32) and make a dynamic niche that could be superior to the exogenous use of cytokines. Jing et al. (2010) showed that the co-culture of mesenchymal stem cells with hematopoietic stem cells could increase the level of hematopoietic cell proliferation compared to the state in which the soluble cytokines added exogenously (33). Some investigations have used the capacity of factors produced by amniotic and chorionic membrane cells to differentiate hUMSCs into germ-like cells $(6,20)$. Asgari et al. showed that co-culture of hUMSCs with placenta cells could increase the expression of OCT4 and DDX4 genes, which indicated the differentiation of hUMSCs into germ-like cells (6). Moreover, AM cells secrete factors such as BDNF, VEGF, HGF, KGF, NT-3, NGF and TGF $\beta$, which play an important role in proliferation, differentiation, plasticity and synapses formation in neurons (34). That's why we used the AM cells for induction of umbilical cord mesenchymal stem cells into neural cells in a co-culture system.

In another experiment, the expression of NSE increased after using bone marrow mesenchymal stem cells alongside growth factors like EGF, HGF and VEGF, while Map2 expression did not alter significantly (35). But in the current study, the expression of both NSE and MAP2 were tripled by co-culturing with AM cells.

The difference in expression of above-mentioned genes can be attributed to two factors: First, the variation in cell type and second, using a co-culture system that causes dynamic secretion of growth factors such as EGF, VEGF and HGF in the culture medium along with other factors that regularly produced in the medium by feeder cells.

On the other hand, dynamic secretion of neuronal growth factors by amniotic membrane cells can simultaneously affect the cells and increase the level of differentiation and expression for MAP2 and NSE genes.

One of the characteristics that distinguish our study is using the proper amount of RA as well as natural inducer and growth factors released by AM cells during the neuronal differentiation process.

In group 4 (RA+ co-culture), the increase in the expression of NSE and MAP2 was quite evident. RA is known as a neuronal pathway inducer, and the presence of RA receptor alpha (RAR $\alpha)(36)$ has been proved to play a major role in neuronal differentiation in mesenchymal stem cells through alteration in proliferation pathway (37).

$\mathrm{Li}$ et al. found that RA could be used as a pre-inducer for the differentiation of mesenchymal stem cells. They revealed that concurrent use of RA combined with modified neuronal medium could increase the differentiation and expression level of both NSE and MAP2 genes in mesenchymal stem cells compared to the modified neural medium alone (36). The results of other research demonstrated that the expression level of NSE increased in the co-culture group of embryonic stem cells with RA, while there was no change in the expression level of ChAT.

Evidently the results of the above studies are consistent with the present study, and in all of them, retinoic acid has been used as a inducer in to neurons, but in this study, an attempt has been made to express more markers with lower doses of acid.

Our Q-PCR data showed that interaction of RA with AM cells could significantly enhance the expression of NSE, MAP2 and ChAT genes. Nonetheless the expression level of ChAT in the group 4 (RA+ co-culture) was higher than both the group 2 and 3 (RA and co-culture groups) alone. Our findings also suggested that co-culturing with AM cells might augment the effect of RA and enhance the optimal expression of neuronal markers.

\section{Conclusion}

Our results demonstrated that hUMSCs can express cholinergic-like cell markers by using co-culture with AM cells and RA as inducer. Using RA and a co-culture system with AM cells can provide proper microenvironment for expression of specific markers and induction of hUMSCs into cholinergic-like cells. Further studies needed to obtain functional cholinergic neurons.

\section{Acknowledgment}

All experiments have been performed at Cellular and Molecular Research Center, IUIMS, Tehran, Iran.

\section{Conflict of Interests}

The authors declare that they have no competing interests.

\section{References}

1. Glotzbach JP, Wong VW, Gurtner GC, Longaker MT. Regenerative medicine. Curr Probl Surg. 2011;48(3):148-212.

2. Raskin J, Cummings J, Hardy J, Schuh K, A Dean R. Neurobiology of Alzheimer's disease: integrated molecular, physiological, anatomical, biomarker, and cognitive dimensions. Curr Alzheimer Res. 2015;12(8):712-22

3. Ma DK, Bonaguidi MA, Ming GL, Song H. Adult neural stem cells in the mammalian central nervous system. Cell Res. 2009;19(6):672-82.

4. Tandon PN, Seth P. Cell therapy for neurological disorders: The elusive goal. Neurol India. 2016;64(4):612-23.

5. Walker T, Huang J, Young K. Neural Stem and Progenitor Cells in 
Nervous System Function and Therapy. Stem Cells Int 2016;2016:1890568.

6. Asgari HR, Akbari M, Abbasi M, Ai J, Korouji M, Aliakbari F, et al. Human Wharton's jelly-derived mesenchymal stem cells express oocyte developmental genes during co-culture with placental cells. Iran J Basic Med Sci. 2015;18(1):22.

7. Alifi F, Asgari HR. Alteration in Expression of Primordial Germ Cell (PGC) Markers During Induction of Human Amniotic Mesenchymal Stem Cells (hAMSCs). JRI. 2020;21(1):59-64.

8. Fong CY, Chak LL, Biswas A, Tan JH, Gauthaman K, Chan WK, et al. Human Wharton's jelly stem cells have unique transcriptome profiles compared to human embryonic stem cells and other mesenchymal stem cells. Stem Cell Rev. 2011;7(1):1-16.

9. Dominici M, Le Blanc K, Mueller I, Slaper-Cortenbach I, Marini F, Krause D, et al. Minimal criteria for defining multipotent mesenchymal stromal cells. The International Society for Cellular Therapy position statement. Cytotherapy. 2006;8(4):315-7.

10. La Rocca G, Anzalone R, Corrao S, Magno F, Loria T, Lo Iacono M, et al. Isolation and characterization of Oct-4+/HLA-G+ mesenchymal stem cells from human umbilical cord matrix: differentiation potential and detection of new markers. Histochem Cell Biol. 2009;131(2):26782.

11. Gauthaman K, Fong CY, Suganya CA, Subramanian A, Biswas A, Choolani M, et al. Extra-embryonic human Wharton's jelly stem cells do not induce tumorigenesis, unlike human embryonic stem cells. Reprod Biomed Online. 2012;24(2):235-46.

12. Sakuragawa N, Kakinuma K, Kikuchi A, Okano H, Uchida S, Kamo I, et al. Human amnion mesenchyme cells express phenotypes of neuroglial progenitor cells. J Neurosci Res. 2004;78(2):208-14.

13. Whittle WL, Gibb W, Challis JR. The characterization of human amnion epithelial and mesenchymal cells: the cellular expression, activity and glucocorticoid regulation of prostaglandin output. Placenta. 2000;21(4):394-401.

14. Koizumi NJ, Inatomi TJ, Sotozono CJ, Fullwood NJ, Quantock AJ, Kinoshita S. Growth factor mRNA and protein in preserved human amniotic membrane. Curr Eye Res. 2000;20(3):173-7.

15. Hass R, Kasper C, Bohm S, Jacobs R. Different populations and sources of human mesenchymal stem cells (MSC): A comparison of adult and neonatal tissue-derived MSC. Cell Commun Signal. 2011;9:12.

16. Uchida $\mathrm{S}$, Inanaga $\mathrm{Y}$, Kobayashi $\mathrm{M}$, Hurukawa $\mathrm{S}$, Araie $\mathrm{M}$, Sakuragawa N. Neurotrophic function of conditioned medium from human amniotic epithelial cells. J Neurosci Res. 2000;62(4):585-90.

17. Ross SA, McCaffery PJ, Drager UC, De Luca LM. Retinoids in embryonal development. Physiol Rev. 2000;80(3):1021-54.

18. Maden M. Retinoic acid in the development, regeneration and maintenance of the nervous system. Nat Rev Neurosci. 2007;8(10):755-65.

19. Li J, Li D, Ju X, Shi Q, Wang D, Wei F. Umbilical cord-derived mesenchymal stem cells retain immunomodulatory and anti-oxidative activities after neural induction. Neural Regen Res. 2012;7(34):266372 .

20. Nejad NA, Amidi F, Hoseini MA, Nia KN, Habibi M, Kajbafzadeh AM, et al. Male germ-like cell differentiation potential of human umbilical cord Wharton's jelly-derived mesenchymal stem cells in coculture with human placenta cells in presence of BMP4 and retinoic acid. Iran J Basic Med Sci. 2015;18(4):325-33.

21. Sun R, Li X, Liu M, Zeng Y, Chen S, Zhang P. Advances in stem cell therapy for cardiovascular disease (Review). Int J Mol Med. 2016;38(1):23-9.

22. Kang JM, Yeon BK, Cho SJ, Suh YH. Stem Cell Therapy for Alzheimer's Disease: A Review of Recent Clinical Trials. J Alzheimers Dis. 2016;54(3):879-89.

23. Ding DC, Chang YH, Shyu WC, Lin SZ. Human umbilical cord mesenchymal stem cells: a new era for stem cell therapy. Cell Transplant. 2015;24(3):339-47.

24. Frausin S, Viventi S, Verga Falzacappa L, Quattromani MJ, Leanza G, Tommasini A, et al. Wharton's jelly derived mesenchymal stromal cells: Biological properties, induction of neuronal phenotype and current applications in neurodegeneration research. Acta Histochem. 2015;117(4-5):329-38.

25. Hu F, Xu P, Sun B, Teng G, Xiao Z. Deep sequencing reveals complex mechanisms of microRNA regulation during retinoic acidinduced neuronal differentiation of mesenchymal stem cells. Genomics. 2017;109(3-4):302-11.
26. Schuldiner M, Eiges R, Eden A, Yanuka O, Itskovitz-Eldor J, Goldstein RS, et al. Induced neuronal differentiation of human embryonic stem cells. Brain Res. 2001;913(2):201-5.

27. Murashov AK, Pak ES, Hendricks WA, Owensby JP, Sierpinski PL, Tatko LM, et al. Directed differentiation of embryonic stem cells into dorsal interneurons. FASEB J. 2005;19(2):252-4.

28. Bahmani L, Taha MF, Javeri A. Coculture with embryonic stem cells improves neural differentiation of adipose tissue-derived stem cells. Neuroscience. 2014;272:229-39.

29. Cui Y, Ma S, Zhang C, Cao W, Liu M, Li D, et al. Human umbilical cord mesenchymal stem cells transplantation improves cognitive function in Alzheimer's disease mice by decreasing oxidative stress and promoting hippocampal neurogenesis. Behav Brain Res. 2017;320:291-301.

30. Marei HES, El-Gamal A, Althani A, Afifi N, Abd-Elmaksoud A, Farag A, et al. Cholinergic and dopaminergic neuronal differentiation of human adipose tissue derived mesenchymal stem cells. J Cell Physiol. 2018;233(2):936-45.

31. Webb S. How retinoic acid makes motor neurons. Nature Reports Stem Cells. 2009.

32. Battiston KG, Cheung JW, Jain D, Santerre JP. Biomaterials in coculture systems: towards optimizing tissue integration and cell signaling within scaffolds. Biomaterials. 2014;35(15):4465-76.

33. Jing D, Fonseca AV, Alakel N, Fierro FA, Muller K, Bornhauser M, et al. Hematopoietic stem cells in co-culture with mesenchymal stromal cells--modeling the niche compartments in vitro. Haematologica. 2010;95(4):542-50.

34. Pardon M-C. Role of neurotrophic factors in behavioral processes: implications for the treatment of psychiatric and neurodegenerative disorders. Vitamins \& Hormones. 8:2: Elsevier; 2010. p. 185-200.

35. Bae KS, Park JB, Kim HS, Kim DS, Park DJ, Kang SJ. Neuron-like differentiation of bone marrow-derived mesenchymal stem cells. Yonsei Med J. 2011;52(3):401-12.

36. Bi Y, Gong M, Zhang X, Zhang X, Jiang W, Zhang Y, et al. Preactivation of retinoid signaling facilitates neuronal differentiation of mesenchymal stem cells. Dev Growth Differ. 2010;52(5):419-31.

37. Janesick A, Wu SC, Blumberg B. Retinoic acid signaling and neuronal differentiation. Cell Mol Life Sci. 2015;72(8):1559-76. 